\title{
Epigenetic consequences of genome manipulations: caveats for human germline therapy and genetically modified organisms
}

\author{
Walter Doerfler*,1 \\ ${ }^{1}$ Institute for Clinical \& Molecular Virology, Friedrich-Alexander University Erlangen-Nürnberg, D-91054 Erlangen \& Institute of \\ Genetics, University of Cologne, D-50674 Cologne, Germany \\ *Author for correspondence: Tel.: +49 171205 1587; walter.doerfler@t-online.de
}

First draft submitted: 13 November 2018; Accepted for publication: 17 January 2019; Published online: 8 February 2019

Keywords: DNA methylation • DNA transfection • epigenetics • foreign DNA in mammalian cells alters epigenetic profiles $\bullet$ genetically modified organisms $\bullet$ gene transcription $\bullet$ human gene therapy $\bullet$ programs for rational decisions $\bullet$ virus infection

The human genome contains about 28 million $\mathrm{CpG}$ dinucleotides that are potential targets for methylation by the DNA methyltransferase system of the cell. There are cell-type-specific patterns of CpG methylation in mammalian and plant systems. Does the intrusion of foreign DNA into mammalian cells affect their DNA CpG methylation and transcription landscapes? This question has been studied in the author's laboratory for many years [1]. The data adduced from different biological systems (viral and human genetic) document significant alterations in these patterns upon entry of foreign DNA into the cell in an episomal or chromosomally integrated configuration. These so far limited investigations call for a broader experimental approach to resolve this issue. Since many research projects in biology, medicine and agriculture involve genome manipulations in mammalian or plant cells and organisms by transfecting foreign DNA with a variety of techniques and a gamut of highly efficient vector systems, the sequelae of these procedures need to be diligently investigated. There is a conspicuous lack of basic experimental work aimed at critically questioning the consequences of genome manipulations for the integrity and functionality of the recipient cells. A comprehensive research program should be developed which investigates the genetic, epigenetic, functional and morphological effects of genome manipulations by using current high throughput technologies. Thus decisions about the acceptability of human gene therapy or genetically modified organisms (GMOs) should be supported by solid scientific evaluations.

The issues raised

The major concern, which the author addresses in this commentary, is the possibility that any manipulations on mammalian or plant cells' genomes and/or the introduction of foreign DNA into these cells might alter the epigenome of the manipulated cell. Neither can we rule out the possibility that merely the employment even of the subtlest techniques, chosen to ferry foreign DNA into cells, might elicit similar responses. The emphasis in this commentary, therefore, is not on familiar mechanisms, like insertional mutagenesis, the haphazard activations of (oncogene) promoters in the vicinity of foreign DNA insertion sites or other traditionally researched mechanisms. The issue here is the possibility that the introduction of foreign DNA might lead to genome-wide alterations of epigenetic signals, in other words, changes in DNA methylation, histone modifications or small RNA activity profiles in genetically modified cells. The consequences of such changes have not been carefully evaluated. There is evidence that DNA methylation and transcription patterns have been affected in investigations of necessarily limited scope carried out over the past 25 years (Table 1) [1]. However, to reach a broader evaluation of epigenetic sequelae caused by foreign DNA entry into mammalian or plant cells, systematic investigations will be called for. With this commentary, I am trying to alert the scientific community to the importance of starting a major coordinated project which aims at evaluating epigenetic consequences upon the intrusion of foreign DNA into mammalian or plant cells. 
Table 1. Genome-wide epigenetic alterations caused by foreign DNA invasions into mammalian cells.

\begin{tabular}{|lllll|}
\hline Mammalian cells & Foreign DNA & Mode of entry & Status inside cells & Ref. \\
\hline BHK 21 hamster cells & Human Ad12 & Ad12 infection & $\begin{array}{l}\text { 12 copies of Ad12 DNA integrated at } \\
\text { one site }\end{array}$ & [2] \\
\hline BHK 21 hamster cells & Bacteriophage lambda & Ca $^{2+}$-phosphate DNA transfection & $\begin{array}{l}\text { Genome integration at one } \\
\text { chromosomal site }\end{array}$ & Genomic integration \\
\hline Human HCT116 cells & p-5.6 kbp bacterial plasmid & DNA nucleofection & Episomal EBV DNA & [5] \\
\hline Human PBMCs & EBV & EBV infection & Genomic integration & Episomal EBV DNA \\
\hline Human PBMCs & Cloned human telomerase gene & Plasmid transfection & Episomal EBV DNA \\
\hline Human lymphoblastoid cell line & EBV & Virus infection & Integrated proviral DNA \\
\hline Human keratinocytes & EBV & Virus infection & Virus infection & [7] \\
\hline Human macrophages & HIV proviral DNA & &
\end{tabular}

Foreign DNA \& manipulations of mammalian or plant genomes Gene therapy

For decades, procedures to modify the genomes of cells and organisms have been established as a widely applied practice. Here I wish to discuss implications for the two most conspicuous fields in which these techniques are employed - human gene therapy and GMOs, the latter both in mammalian and plant cells and organisms. There are reports about the success of these techniques, but caveats remain due to unexpected and possibly unforeseeable consequences. Since the long-term sequelae of the invasion of foreign DNA into cells or organisms have not been extensively studied, projections about the possible problems arising have remained guesswork for far too long. With currently available technologies, at least attempts should be made to alleviate inherent uncertainties.

In frequently used gene therapy of somatic cells - with thousands of clinical trials in progress - the concern of unknown consequences will, in most instances, be prioritized by the intended, sometimes even realized, treatment of disease. Recent reports have documented successful gene editing of the globin genes and the treatment of thalassemia and other hemoglobinopathies by the CRISPR/Cas9 technique [10,11]. However, in many clinical applications, success of gene therapeutic measures cannot be guaranteed, and long-term consequences even of successful curative procedures remain unknown. Gene therapy then constitutes an experimental exercise which is justified in near terminal cases in want of better therapeutic options. There is still much work to be done to determine whether the benefits outweigh the potential risks. There have been fatal accidents in humans, for example, when adenovirus vectors were used [12]. After treating genetically caused immune or other deficiencies, like for example, in patients with Wiskott-Aldrich syndrome, with retroviral vector-linked human genes, rare forms of leukemias have been reported [13]. In some of these instances, the accidental activation of cellular oncogenes by inadvertently placing an inserted gene in the vicinity of the oncogenes' promotors have been invoked as plausible but unproven explanations. Therefore, it is likely that there may be more fundamental problems, like changes of the epigenome, in the wake of foreign DNA insertions that need further consideration and investigation.

A completely different situation arises when cells of the germ line are considered as targets for the insertion of foreign or vector-linked endogenous genes from the same cells or organisms. In germline gene therapy, any genome-wide aftereffects elicited by the introduction of foreign DNA will be transmitted to the offspring with genome-wide epigenetic alterations and incalculable functional manifestations during the organism's lifetime. Most scientists have rightfully recommended a strictly adhered to 'hands-off' attitude toward manipulations on the human germline [14].

\section{Genetically manipulated organisms}

The genetic and epigenetic sequelae of exposing mammalian or plant cells and organisms to foreign DNA, let alone manipulations on a cell's genome, are unknown, because they have not been systematically investigated. Here, one enters into guesswork territory. Researchers who have been active in the perfection of gene technological methods must eventually turn toward critical control studies on the products of their expertise. The successful CRSPR-Cas9 strategies [15] might be a prominent case in point. Methods to conduct these controls have long been available. The recent ruling of the European Court of Justice in Luxembourg [16], that organisms generated by the 'new breeding techniques' have to be considered GMOs, reflects the dilemma that the public and the courts have been left with. 
Box 1. Recommended investigations on genetic and epigenetic consequences upon foreign DNA entry into mammalian cells: comparisons between manipulated and nonmanipulated cells.

1. Select a set of transgenomic organisms/cells - soybean, maize, mice, human cells in culture - which were generated by a multitude of techniques

2. Apply all the known technologies to explore alterations in total genomic sequence, the genome-wide CpG methylation profiles histone modifications, the genome-wide transcriptome, morphological, functional and toxicity studies with transgenomic organisms or cells as compared with nontransgenomic ones. This list of suggestions could be expanded as required

3. Careful and independent statistical analyses of the data adduced in this way

4. An international review board could be selected with members outside the laboratories involved in the experimental work to determine the validity of the experiments

5. Politicians and laboratories, particularly from industry, who hold an interest in the use or production of transgenomic organisms, should be excluded at any stage of this project

In all instances data between manipulated and nonmanipulated controls will have to be compared.

Expert judges, though questionably in biotechnology, have come up with a decision that has puzzled many on either side of the, often ideology or commerce based, opinion spectrum. Granted with limited if any evidence that molecular genetic research has provided on this controversial topic, it would be unfair to blame the members of the court for their far-reaching decision.

On the practical side, which experimental regimen could be proposed to help resolve the highly complex question of whether the dissemination of GMOs will lead the public into harm's way? The perfect answer will not be readily forthcoming. Basic research will be called for to help resolve these issues by rational decisions.

\section{Studies on the epigenetic impact of foreign DNA entry into mammalian cells}

The fate of foreign DNA in mammalian systems $[1,17]$ has been a major interest in our experimental work (Table 1). Hamster cells carrying about 12 copies of chromosomally integrated adenovirus type 12 DNA showed major increases in genome-wide CPG methylation profiles of cellular DNA. These increases remained stable after the complete loss of the adenovirus type 12 genomes [2] supporting the notion that epigenetic alterations in the wake of foreign DNA insertions can be inheritable. Integrated DNA of bacteriophage lambda caused similar changes in the cellular genome, though to a lesser extent [3]. Both types of transgenomic cells were also characterized by altered transcriptional patterns [4]. In a proof-of-principle series of experiments, we documented that the integration of a $5.6 \mathrm{kbp}$ bacterial plasmid, which expressed solely the kanamycin resistance gene to facilitate selection of transgenomic clones, caused significant changes in transcriptional and CpG methylation patterns in the transgenomic as compared with the nontransgenomic human cell clones. Transcription levels were up or downregulated in $4.7 \%$ of the gene segments studied [5]. Several laboratories have shown virus infections to alter the methylation patterns of infected or transformed cells [6-9]. We have concluded that manipulations on a cell's genome can have far-reaching consequences including the generation of completely different cell types $[1,18]$. Our work suggests that a more extensive and proactive study should be initiated to critically evaluate the effects of foreign DNA on the genomes and epigenomes of the recipient cells.

\section{Critical investigations}

International groups of researchers could independently coordinate research along the following lines (Box 1):

This strategy could be a first step toward a more rational discussion on the potential problems inherent in human gene therapy or GMOs in medicine and replace pure guesswork that has dominated this emotionalized debate for decades without reliable conclusions. National Academies of Sciences could take the leadership in organizing and starting projects such as those proposed encompassing additional suggestions, critical analyses and similar or alternate ideas.

Financial \& competing interests disclosure

Research in the author's laboratory has been supported at different times by the Deutsche Forschungsgemeinschaft in Bonn-Bad Godesberg (SFB 74 and 274), the Thyssen Foundation in Cologne (Az. 10.07.2.138), and The Staedtler Stiftung in Nürnberg (WW/eh 01/15). The authors have no other relevant affiliations or financial involvement with any organization or entity with a financial interest in or financial conflict with the subject matter or materials discussed in the manuscript apart from those disclosed.

No writing assistance was utilized in the production of this manuscript. 


\section{References}

Papers of special note have been highlighted as: $\bullet \bullet$ of considerable interest

1. Doerfler W, Weber S, Naumann A. Inheritable epigenetic response towards foreign DNA entry by mammalian host cells: a guardian of genomic stability. Invited review. Epigenetics 13, 1141-1153, (2018).

-. A current summary of the experimental evidence from the author's laboratory demonstrating epigenetic changes in response to the entry of foreign DNA into mammalian cells.

2. Heller H, Kämmer C, Wilgenbus P, Doerfler W. Chromosomal insertion of foreign (adenovirus type 12, plasmid, or bacteriophage lambda) DNA is associated with enhanced methylation of cellular DNA segments. Proc. Natl Acad. Sci. USA 92, 5515-5519 (1995).

-• The first evidence from the author's laboratory linking foreign DNA integration into mammalian genomes and extensive alterations in the DNA methylation profiles of the afflicted cells.

3. Remus R, Kämmer C, Heller H, Schmitz B, Schell G, Doerfler W. Insertion of foreign DNA into an established mammalian genome can alter the methylation of cellular DNA sequences. J. Virol. 73, 1010-1022 (1999).

4. Müller K, Heller H, Doerfler W. Foreign DNA integration. Genome-wide perturbations of methylation and transcription in the recipient genomes. J. Biol. Chem. 276, 14271-14278 (2001).

5. Weber S, Hofmann A, Herms S, Hoffmann P, Doerfler W. Destabilization of the human epigenome: consequences of foreign DNA insertions. Epigenomics 7, 745-755 (2015).

-. A proactive analysis of the epigenetic consequences (altered transcriptional activity and CpG methylation profiles) in human cells transgenomic for a $5.6 \mathrm{kbp}$ bacterial plasmid.

6. Naumann A, Kraus C, Hoogeveen A, Ramirez CM, Doerfler W. Stable DNA methylation boundaries and expanded trinucleotide repeats: role of DNA insertions. J. Mol. Biol. 426, 2554-2566 (2014).

7. Grafodatskaya D, Choufani S, Ferreira JC et al. EBV transformation and cell culturing destabilizes DNA methylation in human lymphoblastoid cell lines. Genomics 95, 73-83 (2010).

-• Destabilization of DNA methylation in human lymphoblastoid cells upon immortalization by EBV.

8. Birdwell CE, Queen KI, Kilgore PC, Trutschl M, Cvek U, Scott RS. Genome-wide DNA methylation as an epigenetic consequence of Epstein-Barr virus infection of immortalized keratinocytes. J. Virol. 88, 11442-11458 (2014).

9. Soto-Girón MJ, Garcia-Vallejo F. Changes in the topology of gene expression networks by human immunodeficiency virus type 1 (HIV-1) integration in macrophages. Virus Res. 163, 91-97 (2012).

10. Dever DP, Bak RO, Reinisch A et al. CRISPR/Cas9 $\beta$-globin gene targeting in human haematopoietic stem cells. Nature 539, 384-389 (2016).

11. Antony JS, Latifi N, Haque AKMA et al. Gene correction of HBB mutations in CD34 $4^{+}$hematopoietic stem cells using Cas9 mRNA and ssODN donors. Mol. Cell Pediatr. 5(1), 9 (2018).

12. Wilson JM. Lessons learned from the gene therapy trial for ornithine transcarbamylase deficiency. Mol. Genet. Metab. 96, 151-157 (2009).

-• A careful analysis of one of the conspicuous failures of human gene therapy.

13. Braun CJ, Witzel M, Paruzynski A et al. Gene therapy for Wiskott-Aldrich syndrome - long-term reconstitution and clinical benefits, but increased risk for leukemogenesis. Rare Dis. 2, (2014).

14. Baltimore D, Berg P, Botchan M et al. A prudent path forward for genomic engineering and germline gene modifications. Science 348, 36-38 (2015).

- The most recent caveat toward germline gene modifications by leaders in the field of molecular genetics.

15. Doudna JA, Charpentier E. Genome editing. The new frontier of genome engineering with CRISPR-Cas9. Science 346 (2014).

-• An important overview of the CRSPR/Cas9 technology and its application to gene editing.

16. Wright AJ. Strict EU ruling on gene-edited crops squeezes science. Nature (2018). https://www.nature.com/articles/d41586-018-07166-7

17. Doerfler W. Foreign DNA in Mammalian Systems. Wiley-VCH, Weinheim, Germany (2000).

18. Doerfler W. Beware of manipulations on the genome: epigenetic destabilization through (foreign) DNA insertions. Epigenomics 8, 587-591 (2016). 